\title{
Finite-amplitude plane waves in ducts with varying properties
}

\author{
Ali Hasan Nayfeh \\ Engineering Science and Mechanics, Virginia Polytechnic Institute and State University, Blacksburg, Virginia 24061 \\ (Received 29 October 1974; revised 23 December 1974) \\ The method of multiple scales is used to determine a first-order uniform expansion for \\ finite-amplitude plane waves of continuous waveforms propagating in a duct having a slowly varying \\ cross section and filled with an inhomogeneous fluid. Losses due to the acoustic boundary layer or a \\ slight wall admittance can be accounted for by decomposing the continuous waveform into its \\ Fourier components and correcting the amplitude and phase of each component independently. Losses \\ at shocks can be accounted for by using weak-shock theory. The results show that the shock \\ formation distance tends to be shortened in a converging section and tends to be lengthened in a \\ diverging section of the duct.
}

Subject Classification: 25.20; 20.45 .

\section{INTRODUCTION}

We consider finite-amplitude plane waves propagating in a duct filled with a fluid which is initially at rest. We assume that the duct cross section, the mean pressure $p_{0}^{*}$, and the mean density $\rho_{0}^{*}$ are slowly varying with axial position.

The problem of finite-amplitude plane waves propagating in a homogeneous bounded or unbounded medium is well understood (see, for example, Blackstock ${ }^{1}$ and Beyer $^{2}$ ).

Recently, the problem of finite-amplitude plane waves propagating in an inhomogeneous medium received considerable attention. Most of this work is concerned with wave propagation in a stratified atmosphere. For example, Randall ${ }^{3}$ and Hayes and Runyan ${ }^{4}$ analyzed the problem of sonic boom, and Einaudi ${ }^{5}$ and Romanova ${ }^{6}$ analyzed finite-amplitude plane waves in an isothermal atmosphere. For more Russian literature, see Ref. 6. The methods used are essentially based on geometrical acoustics accounting for nonlinear distortion or on the assumption that disturbances are given by the linear theory but with a speed of propagation that is the sum of the instantaneous speed of sound and the local convective speed.

A number of approaches have been developed to analyze the linear wave propagation in ducts with variable cross sections (see, for example, the review article by Nayfeh, Kaiser, and Telionis ${ }^{7}$ ). These approaches include expansions for low frequencies, variational methods, expansions for slowly varying cross sections, solutions for slight wall undulations, and approximations of the duct by series of stepped uniform sections.

In this paper, we use the method of multiple scales ${ }^{8}$ to determine a first-order uniform expansion for finiteamplitude plane lossless waves in a duct of slowly varying cross section and filled with an inhomogeneous fluid.

\section{PROBLEM FORMULATION}

We consider finite-amplitude plane waves propagating in a duct with a slowly varying cross section. We assume the fluid to be inviscid, irrotational, and initially quiescent with a nonuniform pressure $p_{0}^{*}$ and a nonuniform density $\rho_{0}^{*}$. We introduce dimensionless quantities using a reference length $x_{r}^{*}$, a reftrence speed $c_{r}^{*}$, and a reference density $\rho_{r}^{*}$. Thus, we let $x=x^{*} / x_{r}^{*}$, $u=u^{*} / c_{r}^{*}, \rho=\rho^{*} / \rho_{r}^{*}, p=p^{*} / \rho_{r}^{*} c_{r}^{* 2}$, and $t=t^{*} c_{r}^{*} / x_{r}^{*}$, where starred and unstarred quantities denote dimensional and dimensionless quantities, respectively, $x$ is the axial position, $u$ is the axial velocity, $\rho$ is the fluid density, $p$ is the fluid pressure, and $t$ is the time. In terms of these dimensionless quantities and the dimensionless cross section $A=A^{*} / A_{r}^{*}$ of the duct, the quasi-one-dimensional equations describing the conservation of mass and momentum are

$$
\begin{aligned}
& \frac{D \rho}{D t}+\frac{\rho}{A} \frac{\partial}{\partial x}(u A)=0, \\
& \frac{D u}{D t}=-\frac{1}{\rho} \frac{\partial p}{\partial x}+g,
\end{aligned}
$$

where $g$ is a constant body acceleration and

$$
\frac{D}{D t}=\frac{\partial}{\partial t}+u \frac{\partial}{\partial x} \text {. }
$$

For an inviscid irrotational motion, the conservation of energy yields

$$
D S / D t=0 \text {, }
$$

where $S$ is the entropy. Hence,

$$
\frac{D p}{D t}=c^{2} \frac{D \rho}{D t}
$$

To determine an equation for $u$, we form $D / D t$ of Eq. 2, use Eq. 4, and obtain

$$
\frac{D^{2} u}{D t^{2}}=\frac{1}{\rho^{2}} \frac{D_{\rho}}{D t} \frac{\partial p}{\partial x}-\frac{1}{\rho} \frac{\partial}{\partial x}\left(c^{2} \frac{D_{\rho}}{D t}\right)+\frac{1}{\rho} \frac{\partial u}{\partial x} \frac{\partial p}{\partial x} .
$$

Substituting for $D \rho / D t$ from Eq. 1 into Eq. 5 and arranging, we have

$$
\begin{aligned}
\frac{1}{c^{2}} \frac{D^{2} u}{D t^{2}}= & \frac{\partial^{2} u}{\partial x^{2}}+\frac{\partial}{\partial x}\left[\ln \left(\rho c^{2} A\right)\right] \frac{\partial u}{\partial x}+u\left\{\frac{d^{2}}{d x^{2}}(\ln A)\right. \\
& \left.+\frac{d}{d x}(\ln A)\left[\frac{\partial}{\partial x}\left(\ln \rho c^{2}\right)-\frac{1}{\rho c^{2}} \frac{\partial p}{\partial x}\right]\right\} .
\end{aligned}
$$

To determine an approximate solution to Eqs. 1 and 6, we assume that the initial density $\rho_{0}$, the initial pressure $p_{0}$, and the cross section $A$ are functions of a slow scale $x_{1}=\epsilon x$, where $\epsilon$ is a small dimensionless parameter. Moreover, we assume that $p-p_{0}$ is an analytic 
function of the condensation ${ }^{2}$

$$
s=\left(\rho / \rho_{0}\right)-1,
$$

so that

$$
c^{2}=c_{0}^{2}\left(1+c_{1} s+c_{2} s^{2}+\cdots\right),
$$

where the $c_{n}$ are known functions of $x_{1}$. Substituting for $\rho$ and $c^{2}$ from Eqs. 7 and 8 into Eqs. 1 and 6 and subtracting the mean parts, we obtain

$$
\begin{aligned}
\frac{\partial s}{\partial t}+\frac{\partial u}{\partial x}=-\epsilon u(1+s) \frac{d}{d x_{1}}\left[\ln \left(\rho_{0} A\right)\right]-\frac{\partial}{\partial x}(s u), & \\
\frac{\partial^{2} u}{\partial x^{2}}-\frac{1}{c_{0}^{2}} \frac{\partial^{2} u}{\partial t^{2}}= & \frac{1}{c_{0}^{2}}\left[-c_{1} s \frac{\partial^{2} u}{\partial t^{2}}+\frac{\partial}{\partial t}\left(u \frac{\partial u}{\partial x}\right)+u \frac{\partial^{2} u}{\partial x \partial t}\right] \\
& \quad-\epsilon \frac{d}{d x_{1}}\left[\ln \left(\rho_{0} c_{0}^{2} A\right)\right] \frac{\partial u}{\partial x}-\left(1+c_{1}\right) \frac{\partial s}{\partial x} \frac{\partial u}{\partial x}+O\left(\epsilon^{3}\right),
\end{aligned}
$$

where $u$ and $s$ are assumed to be $O(\epsilon)$.

\section{AN APPROXIMATE SOLUTION}

To determine an approximate solution to Eqs. 9 and 10 , we use the method of multiple scales and assume that $u$ and $s$ have expansions of the form

$$
\begin{aligned}
& u=\epsilon u_{1}\left(\xi, \eta, x_{1}\right)+\epsilon^{2} u_{2}\left(\xi, \eta, x_{1}\right)+\cdots, \\
& s=\epsilon s_{1}\left(\xi, \eta, x_{1}\right)+\epsilon^{2} s_{2}\left(\xi, \eta, x_{1}\right)+\cdots,
\end{aligned}
$$

where

$$
\xi=t-\int \frac{d x}{c_{0}}, \eta=t+\int \frac{d x}{c_{0}} .
$$

The temporal and spatial derivatives transform according to

$$
\begin{aligned}
\frac{\partial}{\partial t}= & \frac{\partial}{\partial \xi}+\frac{\partial}{\partial \eta}, \\
\frac{\partial^{2}}{\partial t^{2}}= & \frac{\partial^{2}}{\partial \eta^{2}}+\frac{\partial^{2}}{\partial \xi^{2}}+2 \frac{\partial^{2}}{\partial \xi \partial \eta}, \\
\frac{\partial}{\partial x}= & \frac{1}{c_{0}}\left(\frac{\partial}{\partial \eta}-\frac{\partial}{\partial \xi}\right)+\frac{\partial}{\partial x_{1}}, \\
\frac{\partial^{2}}{\partial x^{2}} & =\frac{1}{c_{0}^{2}}\left(\frac{\partial^{2}}{\partial \eta^{2}}-2 \frac{\partial^{2}}{\partial \xi \partial \eta}+\frac{\partial^{2}}{\partial \xi^{2}}\right)+\frac{2 \epsilon}{c_{0}}\left(\frac{\partial^{2}}{\partial \eta \partial x_{1}}-\frac{\partial^{2}}{\partial \xi \partial x_{1}}\right) \\
& +\frac{\epsilon c_{0}^{\prime}}{c_{0}^{2}}\left(\frac{\partial}{\partial \xi}-\frac{\partial}{\partial \eta}\right)+\epsilon^{2} \frac{\partial^{2}}{\partial x_{1}^{2}},
\end{aligned}
$$

where primes denote differentiation with respect to the argument.

Substituting Eqs. 11-17 into Eqs. 9 and 10 and equating the coefficients of $\epsilon$ on both sides, we obtain

$$
\begin{aligned}
& \frac{\partial s_{1}}{\partial \xi}+\frac{\partial s_{1}}{\partial \eta}+\frac{1}{c_{0}}\left(\frac{\partial u_{1}}{\partial \eta}-\frac{\partial u_{1}}{\partial \xi}\right)=0, \\
& -\frac{4}{c_{0}^{2}} \frac{\partial^{2} u_{1}}{\partial \xi^{\partial} \eta}=0 .
\end{aligned}
$$

The solutions of Eqs. 18 and 19 are taken in the form of a wave traveling in the positive direction; that is, we let

$$
u_{1}=g\left(\xi, x_{1}\right), s_{1}=c_{0}^{-1} g\left(\xi, x_{1}\right) .
$$

Substituting Eqs. 11-17 into Eq. 10, equating the coefficients of $\epsilon^{2}$ on both sides, and using Eq. 20, we have

$$
\begin{aligned}
-\frac{4}{c_{0}^{2}} \frac{\partial^{2} u_{2}}{\partial \xi \partial \eta}= & -\frac{c_{1}+2}{c_{0}^{3}} \frac{\partial}{\partial \xi}\left[g \frac{\partial g}{\partial \xi}\right]-\frac{d}{d x_{1}}\left[\ln \left(\rho_{0} c_{0}^{2} A\right)\right] \frac{\partial g}{\partial \xi} \\
& +\frac{2}{c_{0}} \frac{\partial^{2} g}{\partial \xi \partial x_{1}}-\frac{c_{0}^{\prime}}{c_{0}^{2}} \frac{\partial g}{\partial \xi} .
\end{aligned}
$$

Since $g=g\left(\xi, x_{1}\right)$, the particular solution of Eq. 21 contains a term proportional to $\eta$, making $\left|\epsilon^{2} u_{2}\right|$ larger than $\left|\epsilon u_{1}\right|$ for either large $t$ or large $x$, contrary to the assumption under which the expansion is obtained. For a uniform expansion, the right-hand side of Eq. 21 must vanish; that is,

$$
\begin{aligned}
-\frac{c_{1}+2}{c_{0}^{3}} \frac{\partial}{\partial \xi}\left[g \frac{\partial g}{\partial \xi}\right] & +\frac{d}{d x_{1}}\left[\ln \left(\rho_{0} c_{0}^{2} A\right)\right] \frac{\partial g}{\partial \xi} \\
& +\frac{2}{c_{0}} \frac{\partial^{2} g}{\partial \xi \partial x_{1}}-\frac{c_{0}^{\prime}}{c_{0}^{2}} \frac{\partial g}{\partial \xi}=0 .
\end{aligned}
$$

This equation is satisfied by its first integral,

$$
\frac{\partial g}{\partial x_{1}}+\frac{d}{d x_{1}}\left[\ln \left(\rho_{0} c_{0} A\right)^{1 / 2}\right] g-\frac{\left(c_{1}+2\right)}{2 c_{0}^{2}} g \frac{\partial g}{\partial \xi}=0 \text {. }
$$

It is convenient to transform Eq. 23 into an equation with constant coefficients. To this end, we let

$$
g=\psi\left(x_{1}\right) G\left(\xi, x_{1}\right), \quad z=\phi\left(x_{1}\right)
$$

in Eq. 23 and obtain

$\frac{\partial G}{\partial z}+\frac{1}{\phi^{\prime}}\left\{\frac{\psi^{\prime}}{\psi}+\frac{d}{d x_{1}}\left[\ln \left(\rho_{0} c_{0} A\right)^{1 / 2}\right]\right\} G-\frac{\left(c_{1}+2\right) \psi}{2 c_{0}^{2} \phi^{\prime}} G \frac{\partial G}{\partial \xi}=0$.

We choose

$$
\begin{aligned}
& \frac{\psi^{\prime}}{\psi}+\frac{d}{d x_{1}}\left[\ln \left(\rho_{0} c_{0} A\right)^{1 / 2}\right]=0, \\
& \phi^{\prime}=\frac{\left(c_{1}+2\right) \psi}{2 c_{0}^{2}},
\end{aligned}
$$

so that Eq. 25 becomes

$$
\frac{\partial G}{\partial z}-G \frac{\partial G}{\partial \xi}=0 \text {. }
$$

Equation 26 is satisfied by

$$
\psi=\left(\rho_{0} c_{0} A\right)^{-1 / 2} .
$$

Then, the solution of Eq. 27 can be written as

$$
\phi=\int \frac{c_{1}+2}{2 c_{0}^{2}\left(\rho_{0} c_{0} A\right)^{1 / 2}} d x_{1} .
$$

Moreover, the solution of Eq. 28 is

$$
G=f(\xi+z f) \text {. }
$$

Eliminating $G, \phi$, and $\psi$ from Eqs. 24, 29, and 30, setting $u_{1}=g$, and substituting the result into Eq. 11, we obtain

$$
\begin{aligned}
u= & \epsilon\left(\rho_{0} c_{0} A\right)^{-1 / 2} f\left[t-\int_{x_{r}}^{x} \frac{d x}{c_{0}}+\epsilon f \int_{x_{r}}^{x} \frac{c_{1}+2}{2 c_{0}^{2}\left(\rho_{0} c_{0} A\right)^{1 / 2}} d x\right] \\
& +O\left(\epsilon^{2}\right) .
\end{aligned}
$$

In the case of waves propagating in a uniform duct filled with a fluid having uniform properties, $\rho_{0}=c_{0}=A=1$ and Eq. 32 reduces to

$$
u=\epsilon f\left[t-x+\frac{1}{2} \epsilon\left(c_{1}+2\right) x f\right]+O\left(\epsilon^{2}\right),
$$


which is the familiar solution for the case of finite-amplitude plane waves propagating in a homogeneous medium. ${ }^{1}$ The solution for the case of waves propagating in a uniform duct filled with an inhomogeneous fluid or in an unbounded inhomogeneous fluid can be obtained by letting $A=1$ in Eq. 32. The result is in agreement with that obtained independently by Carlton and Blackstock ${ }^{12}$ by using a different method of solution.

\section{CONCLUDING REMARKS}

The solution consisting of Eq. 32 becomes multivalued at some distance $x=l$, as a result of steepening of the waveform, and is therefore invalid beyond that distance because pressure disturbances cannot be multivalued, either in space or in time. Physically, a shock wave begins to form and viscous effects cannot be neglected. This constitutes a major limitation of the inviscid, irrotational flow assumption. To continue the solution farther, viscous effects must be included in the analysis. This can be accomplished by including the viscous terms in the original equations and deriving a Burgers' equation or by using weak-shock theory. ${ }^{1}$ Equation 32 shows that the amplitude of the wave increases if the duct converges, leading to a shortening of the shock formation distance. In a diverging section of the duct, the amplitude of the wave decreases thereby increasing the shock formation distance.

Although viscous effects can be neglected in the body of the fluid away from any shocks, they cannot be neglected near the duct walls. These viscous effects, although small, tend to significantly distort the waves over long distances by dispersing and attenuating the individual frequency components. ${ }^{9,10}$ Following Cook ${ }^{11}$ and Pestorius and Blackstock, ${ }^{10}$ one can account for these viscous effects as well as the effects of a small wall admittance by decomposing the continuous waveform into its Fourier components and modifying the complex amplitude of each component individually. To do this, we note that the effective wall admittance for plane waves in a perfect gas is ${ }^{7}$

$$
\beta_{e}=\beta+(1-i) \alpha, \quad \alpha=\left(\frac{\omega}{2 \operatorname{Re}}\right)^{1 / 2}\left[1+\frac{\gamma-1}{(\operatorname{Pr})^{1 / 2}}\right],
$$

where $\operatorname{Re}=\rho_{r} c_{r} x_{r} / \mu, \gamma$ is the specific heat ratio of the gas, $\operatorname{Pr}$ is the Prandtl number, and $\mu$ is the coefficient of viscosity. Thus, the complex amplitude of a Fourier component of frequency $\omega$ is multiplied by $\exp [-\beta \Delta x$ $-(1-i) \alpha \Delta x]$ to account for the distortion of the waveform over a short distance $\Delta_{x}$ due to boundary-layer and wall admittance effects.
We stress the fact that the present analysis is valid for slowly varying cross sections and mean flow quantities. This condition implies that $A^{\prime}(x) / A(x), \rho_{0}^{\prime}(x) / \rho_{0}(x)$, and $c_{i}^{\prime}(x) / c_{i}(x)$ are of the order of the perturbation parameter $\epsilon$ and hence are small. Since the expansion is carried out to $O(\epsilon)$, the error in Eq. 32 is $O\left(\epsilon^{2}\right)$. For example, for a duct with a flare of $6^{\circ}$, the error in Eq. 32 is $O(1 \%)$. As the flare angle increases, the error increases.

\section{ACKNOWLEDGMENTS}

The author is grateful to Dr. D. T. Blackstock for pointing out that in an inhomogeneous medium the entropy may vary in the medium although the entropy of an individual fluid particle stays constant during the motion.

${ }^{1}$ D. T. Blackstock, "Nonlinear Acoustics (Theoretical)," in American Institute of Physics Handbook, D. E. Gray, Ed. (McGraw-Hill, New York, 1972), 3-183-3-205.

${ }^{2}$ R. T. Beyer, "Nonlinear Acoustics (Experimental)," in American Institute of Physics Handbook, D. E. Gray, Ed. (McGraw-Hill, New York, 1972), 3-206-3-210.

${ }^{3}$ D. G. Randall, "Sonic Bang Intensities in a Stratified, Still Atmosphere," J. Sound Vib. 8, 196-214 (1968).

${ }^{4}$ W. D. Hayes and H. L. Runyan Jr., "Sonic-Boom Propagation Through a Stratified Atmosphere," J. Acoust. Soc. Am. 51, 695-701 (1970).

${ }^{5}$ F. Einaudi, "Singular Perturbation Analysis of Acoustic-Gravity Waves," Phys. Fluids 12, 752-756 (1969).

${ }^{6}$ N. N. Romanova, "The Vertical Propagation of Short Acoustic Waves in the Real Atmosphere," Izv. Atm. Ocean Phys. 6, 134-145 (1970) (English transl., pp. 73-78).

${ }^{7}$ A. H. Nayfeh, J. E. Kaiser, and D. P. Telionis, "The Acoustics of Aircraft Engine-Duct Systems," AIAA J. 13, 130-153 (1975).

${ }^{8}$ A. H. Nayfeh, Perturbation Methods (Wiley-Interscience, New York, 1973), Chap. 6.

${ }^{9}$ A. B. Coppens, "Theoretical Study of Finite-Amplitude Traveling Waves in Rigid-Walled Ducts: Behavior for Strengths Precluding Shock Formation," J. Acoust. Soc. Am. 49, 306318 (1971).

${ }^{10}$ F. M. Pestorius and D. T. Blackstock, "Nonlinear Distortion in the Propagation of Intense Acoustic Noise," in Interagency Symposium on University Research in Transportation Noise, Vol. II (Stanford U. P., Stanford, 1973), pp. 565-577.

${ }^{11}$ B. D. Cook, "New Procedure for Computing Finite-Amplitude Distortion," J. Acoust. Soc. Am. 34, 941-946 (1962).

${ }^{12}$ T. W. Carlton and D. T. Blackstock, "Propagation of Plane Waves of Finite Amplitude in Inhomogeneous Media with Applications to Vertical Propagation in the Ocean," ARL-TR-7431, Univ. Texas at Austin, 22 Aug. 1974; see also J. Acoust. Soc. Am. 56, S42 (A) (1974). 